\title{
Technologies based on energy savings for OLED devices
}

\author{
Mohammed Albaba ${ }^{1, *}$, Meryem Sena Akkus ${ }^{2}$ \\ 1,* Electrical and Electronics Engineering Department, Ankara Yildirim Beyazit University, Ankara/Turkey, \\ 195105403@ybu.edu.tr, ORCID: 0000-0002-0833-8191 \\ ${ }^{2}$ Central Research Laboratory Application and Research Center, Ankara Yildirim Beyazit University, Ankara/Turkey, \\ msakkus@ybu.edu.tr, ORCID: 0000-0003-2550-550X
}

\section{A B S T R A C T}

Recently, scientists are paying more attention to the Organic Light Emitting Diode (OLED) technology as it is being used in devices and displays to play videos and show photos with high resolution. This technology is used in products such as mobile phones, televisions, laptops, etc. To make the energy consumed less, new methods were shown up to prevent high energy consumption while presenting videos and photos on OLED devices and displays without losing their details and quality, one of the methods is a deep learning-based technique which is related to artificial intelligence. In this review paper, the last methods were discussed as well as their results. Saturation, brightness, contrast, and luminance are factors that impacting energy consumption. In terms of OLED mobile phones, there were a few studies that concentrated on turning off the unnecessary pixels which will be black as default, and as a result, the lifetime of batteries will be extended. Also, for OLED mobile phones, a web browser called Chameleon was presented as it has some modes to save the energy consumed while surfing the internet by remapping the displayed colors of the website.

\section{ARTICLE INFO}

Research article

Received: 2.02.2021

Accepted: 25.06.2021

Keywords:

OLED Displays,

Power Consumption,

Deep Learning Method,

Chameleon Web

Browser,

Reducing Power

${ }^{*}$ Corresponding author

\section{Introduction}

Organic Light-Emitting Diode (OLED) display is a new technology that showed better results than liquid crystal displays (LCD) in terms of brightness, good view, and the ability to make flexible displays from it [1]. In the last few years, a lot of display industries started to merge OLED displays in their products such as smartphones, TVs, wearable devices, and much more. Also, transparent OLED display (TOLED) was presented lately, as many companies started to merge it in their goods like military products, vehicle construction, and other industries [2]. To decrease the power consumption in OLED displays, some methods were made in terms of hardware enhancement and image processing [3,4,5]. This review paper talks about the next methods, Unsupervised PCCE Technique, Region Of Interest (ROI) Extraction \& Region Of Noninterest (NON-ROI) Dimming, A Web Browser For Mobile OLED Displays; Chameleon, and Darkening OLED Display Partially, that were used to achieve the desired goal. In terms of mobile devices, it was found out that screens consuming the highest ratio of the battery's power by comparing it with other components with a percentage between 38-50\% [6,7]. The advantage of OLED displays is that each pixel emits light by itself without needing a backlight, unlike LCDs. Therefore, it easier to save power consumed by turning off the unnecessary pixel according to the displayed image or video as every pixel emits the green, red and blue color [8, 9]. According to Dong and Zhong, the power consumption is low in black color and highest in white color $[10,11]$. Some researchers advised that changing color sets may affect the energy consumed by OLED and make it less by $40 \%$ [12].

In the experience of web browsing using smartphones with OLED displays, it is known that webpages have different color designs, especially white color which is 80\% [13], some of them use GIFs and flash videos which increase the power demand from the battery to display them on OLED displays. Mobile devices that have OLED displays are facing some problems in terms of energy saving and researchers say that losing energy could be less when the reduction of brightness is applied, but the user will not be satisfied [14]. Some methods were implemented to reduce power consumption while keeping and preserving the details of images displayed on OLED display. One of the methods is depending on the power-constrained contrast enhancement (PCCE) implemented by using one of deep learning algorithms [15]. Another method is by darkening the image by reducing the luminance of some pixels according to the displayed image on OLED display, in other words, remapping the colors of the image [8]. One more method depended on the dark scheme 
which generates a part of black color on OLED screen with a specific percentage [16].

\section{Methods and Implementations}

In this section, some methods will be explained in terms of saving power while preserving the displayed details of the image according to some studies. Also, other studies will be explained on mobile devices that use the OLED display.

\subsection{Unsupervised PCCE Technique}

This method relates to one of deep learning algorithms directly that called Convolutional Neural Network (CNN). Deep learning outperforms the other methods in terms of image enhancements $[17,18]$. It is used to enhance power consumption by reducing it while viewing the most improved photo in terms of brightness and details on an OLED display [15]. This display's total dissipated power (TDP) that has N pixels is expressed according to $[4,19,20]$ as follows:

$$
\mathrm{P}=\sum_{i=1}^{N}\left(\mathrm{w}_{o}+\mathrm{w}_{R} \mathrm{R}_{i}^{\gamma}+\mathrm{w}_{G} \mathrm{G}_{i}^{\gamma}+\mathrm{w}_{B} \mathrm{~B}_{i}^{\gamma}\right)
$$

Whereas $R_{i}, G_{i}$ and $B_{i}$ are the intensities of red, green and blue color for the $i$ pixel, wo constant value of power giving to for the non-pixel section of display, $W_{R}, W_{G}, W_{B}$ and $\gamma$ are the values of coefficients that are depending on the panel.

This method requires a dataset to be implemented, the dataset was chosen to be Berkeley Segmentation Dataset (BSD) [21]. To train a deep learning CNN model, two components must be considered which are the network architecture design and the loss function. Context aggregation network (CAN) [22] was employed as the base network because it has the feature of demanding a small number of network parameters. For the loss function, training without the reference image was made as it is related to unsupervised learning. By doing this, the enhancement of image's contrast is applied, and at the same time, power consumption is being saved [15]. The method divides the image into two images, luminance, and color. The luminance's intensity reduces to decrease the power demand, of course, the contrast in this way improved by using the deep learning method, CNN, and after that, the resulted photo will be added to the color image as shown in Figure 1 [15].

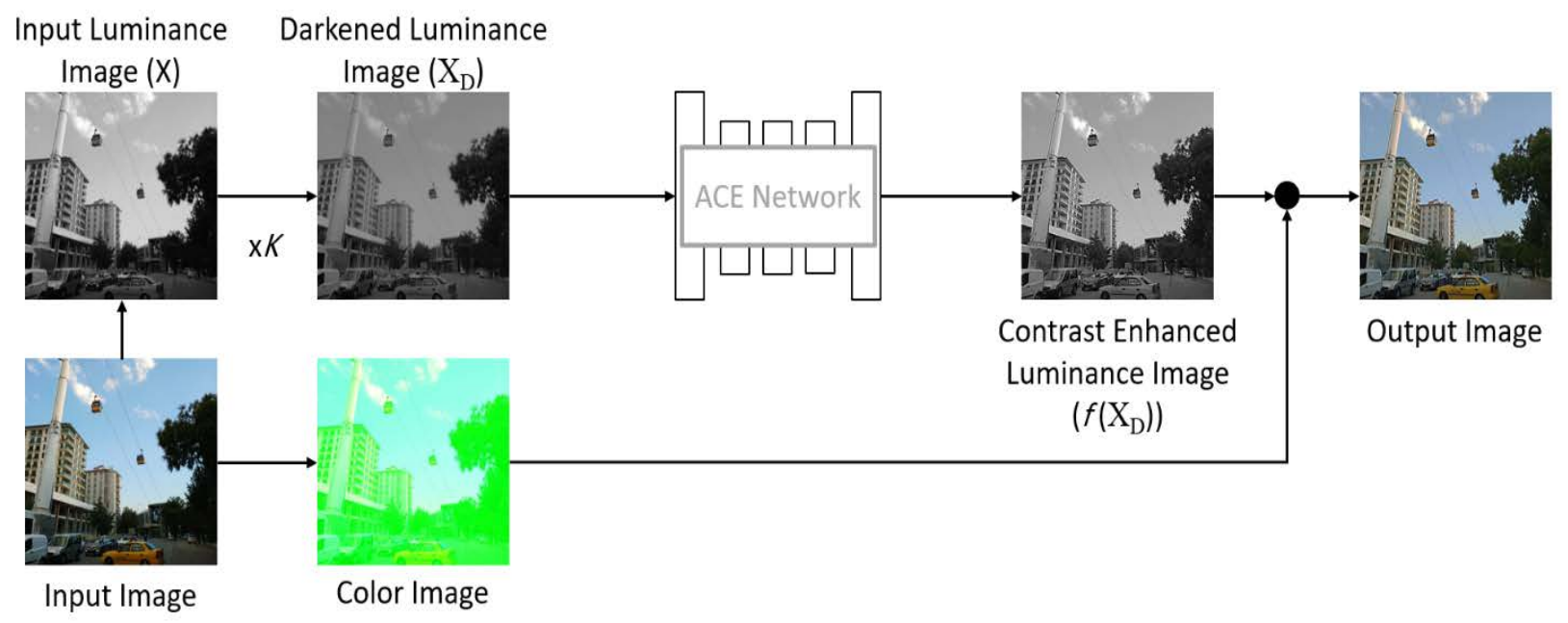

Figure 1. The image before and after implementing CNN method [Adapted from reference 15].

After reducing the luminance of the input image, the rate of power saving (R) will be reduced as well as shown in Eq. (2). $\mathrm{P}_{\text {dim }}$ and $\mathrm{P}_{\text {in }}$ are TDP of dimmed and input images, and $\mathrm{k}$ is a ratio of reducing the luminance image's intensity. By implementing this, the image quality may reduce and lose its details, for that the adaptive contrast enhancement (ACE) was introduced to improve the contrast of darkening image [15].

$$
\mathrm{R}=1-\frac{P_{\operatorname{dim}}}{P_{\text {in }}}=1-\frac{\sum_{i}^{N}\left(k Y_{i}\right)^{\gamma}}{\sum_{i}^{N}\left(Y_{i}\right)^{\gamma}}=1-k^{\gamma}
$$

Whereas $R_{i}, G_{i}$, and $B_{i}$ are the intensities of red, green, and blue color for the $i$ pixel, Wo constant value of power giving to for the non-pixel section of the display, $\mathrm{W}_{\mathrm{R}}, \mathrm{W}_{\mathrm{G}}, \mathrm{W}_{\mathrm{B}}$, and $\gamma$ are the values of coefficients that are depending on the panel.

As shown in Figure 2, the ACE network architecture contains some layers from the input image which is $\mathrm{L}_{0}$ to the output image that is $\mathrm{L}_{\mathrm{d}}$. Through the process, the image is being processed by applying the CNN method on it to reach the best image details with less power demand [15]. 


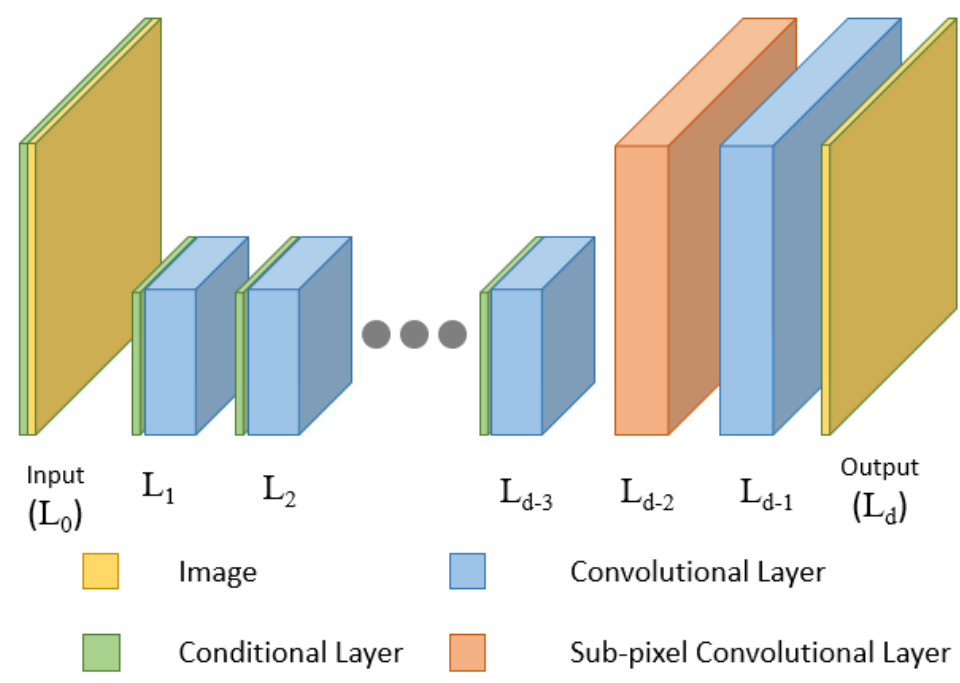

Figure 2. The ACE network architecture [Adapted from reference 15].

In terms of the loss function, there are three factors that make the total loss function which is power loss $\mathrm{L}_{\mathrm{p}}$, similarity loss $L_{s}$ and contrast loss $L_{c}$. The first factor makes sure that the power is reduced after improving contrast, the second factor sets the changing of the image, and the third factor is enhancing the output image's contrast. The sum of the three losses is giving total loss as it is illustrated in Eq. 3. [15]. Hyper parameters that control-related importance to every factor is $\lambda_{\mathrm{p}}, \lambda_{\mathrm{s}}$, and $\lambda_{\mathrm{C}}$.

$$
\mathrm{L}_{\text {total }}=\lambda_{P} L_{P}+\lambda_{s} L_{s}+\lambda_{c} L_{c}
$$

For training the ACE network, 300k iterations were trained with a 0.0001 learning rate [23, 24]. Adam optimizer was used as well [25]. The ACE network has seven layers, and the rate of power-saving $(\mathrm{R})$ was between $[0.01,0.8]$ for every image in the training section for every iteration, while in the test section, the range was between [0.1 - 0.7] with a step of 0.1 .

The process is done by using a single Titan X GPU with the usage of Tensorflow. The dataset is the BSDS500 dataset [21] which has 500 images, it is randomly separated into the training section and the testing section as 400 images and 100 images, respectively. As a result, the training process is good and it is done without the problem of over-fitting.

In terms of video displaying, an experiment was applied for two movies which are 'The Shawshank Redemption' and 'Avatar' [26]. An average luminance with the value of $\mathrm{R}$ in the ACE network was employed for the frame of the input video. In dark scenes, the R-value is approximately 0 to stop reducing brightness, whereas it has a large value for bright scenes and frames to reduce their brightness in order to reduce power consumption [15].

\subsection{Region Of Interest (ROI) Extraction \& Region Of Noninterest (NON-ROI) Dimming}

Here, by using extraction and dimming features and applying them to the image, the power consumption reduces. In addition, the details of the image save as well.

Dong \& Zhong were the first who modeled the formulas for optimizing and modeling power for the OLED displays, they designed Eq. 4 which shows the power contributed for one pixel in terms of three colors are red, green, and blue, whereas $f(R), h(G)$, and $k(B)$ are expressing the consumed power for each color in the pixel; red, green and blue, respectively.

Eq. 5 shows the full power consumption that the OLED display consumes for $n$ pixels. There is a constant value which is $\mathrm{C}$ that is expressing the stable power consumption required by control chips [10].

$$
\begin{aligned}
& P_{\text {pixel }}(R, G, B)=f(R)+h(G)+k(B) \\
& P=C+\sum_{i=1}^{n}\left\{f\left(R_{i}\right)+h\left(G_{i}\right)+k\left(B_{i}\right)\right\}
\end{aligned}
$$

It depends on getting rid of the un-useful details while keeping the area of interest in the photo. It has two levels; the first level is extracting the region of interest (ROI) by using the detection model on the photo. The second level is by changing the saturation and luminance degrees that are related to the noninterest region (NON-ROI) of the photo. By implementing this technique, the consumed power reduces as the NON-ROI is getting dimmed by changing its parameters which are saturation and luminance. 
To use the abstraction method on ROI, the Canny [27] algorithm was used, also, and for changing luminance and saturation values for NON-ROI, ALS (adjusting luminance \& saturation) method was used.

In terms of ROI, the Canny method is designed for a gray image to find the edges of it and because of the improvements of this industry, it uses to detect the edges of color photos [27]. So, to apply this method, by using Eq. 6, the photo converts to gray [8].

$$
P_{\text {Gray }}=P_{R} * 0.299+P_{G} * 0.587+P_{B} * 0.114
$$

After that, the resulted gray photo will be smoothed. Then, the areas of images with high and sharp gray intensity will be found, and a gradient will be applied to them, this step is called non-maximum suppression. Then after, and to get rid of the noise in the photo, it is a substantial thing to filter the undesired low gradient value. Finally, the pixels will be connected to each other forming the edges.

In terms of NON-ROI, and by changing the values of saturation and luminance, the power consumption will be reduced. Keeping the quality and details of the photo are important in this case. These factors will be applied to NONROI related to the photo to make sure that the photo still has almost the same requirements for the user vision.

For changing luminance value, it is known that the pixel's luminance is related to the red, green, and blue colors. When changing pixel's luminance, their values reduce power consumption. The luminance for a pixel is the sum of values of R, G, and B colors for a pixel, Eq. 7 shows the function of sum. Adjustment of luminance is expressed by Eq. 8. $\mathrm{P}_{\text {pixel }}$ is prime luminance for one pixel, whereas $\mathrm{P}_{\text {pixel }}$ is expressing luminance after adjusting it. The parameter of adjusting is $\mathrm{X}$ which has a value between $\{0-1\}$. Adjustment regions' number in the noninterest region in the image is expressed by $\mathrm{N}$. Region number of pixels located is $\mathrm{n}_{\mathrm{i}}$.

$$
\begin{gathered}
P_{\text {pixel }}(S)=f(R, G, B) \\
P_{\text {pixel }}^{\prime}(S)=f(R, G, B) *\left(1-X *\left(\frac{n_{i}}{N}\right)\right)
\end{gathered}
$$

To change the value of saturation, and according to OLED displays, the blue color component was found that it is consuming higher power than other color components as shown in Figure 3. So, when adjusting the saturation value of the photo, it means that the values of the components that consuming higher power will be reduced and the bright image will be dimmed as well. The method's name is Adjusting Luminance \& Saturation (ALS) [8]. For videos and mobile games, the darkening method is a good technique to be implemented in order to save more energy [28, 29].

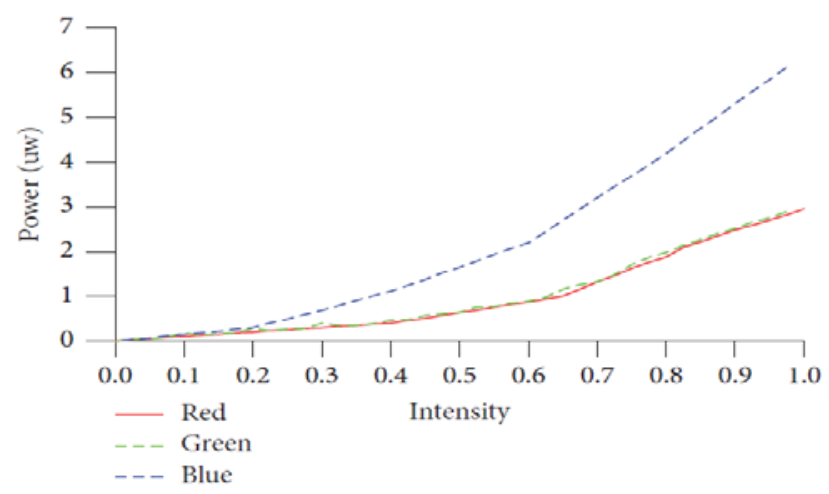

Figure 3. Power consumed by red, green, and blue components of OLED pixel [Reproduced with permission from reference 8. Copyright 2016, Deguang Li et al. Mobile information systems].

\subsection{A Web Browser For Mobile OLED Displays; Chameleon}

In terms of internet usage and surfing various websites on mobile OLED displays, a web browser color-adaptive is presented. OLED displays are consuming a lot of energy when the main color is white, which is a power-hungry color. It is obvious that the black color consumes much less power.

For one pixel $I$, the total power consumption is expressed by Eq. 9, meanwhile, Eq. 10 is to calculate the power consumption for an OLED display of $N$ pixels [30].

$$
\begin{gathered}
P_{i}=a \cdot R_{i}+b \cdot G_{i}+c \cdot B_{i} \\
P=\sum_{i=1}^{N} P_{i}=\sum_{i=1}^{N}\left(a \cdot R_{i}+b \cdot G_{i}+c \cdot B_{i}\right)
\end{gathered}
$$

As it is noticeable, most of websites are depending on white color in their color design for displaying their websites' contents. From that point, the Chameleon web browser raised up to recolor the GUI objects of websites, while keeping images without changes. The GUI objects' colors are being transformed by counting, mapping, and painting them again in a way that keeps the consistency of their colors for each website as is illustrated in Figure 4 [31]. 


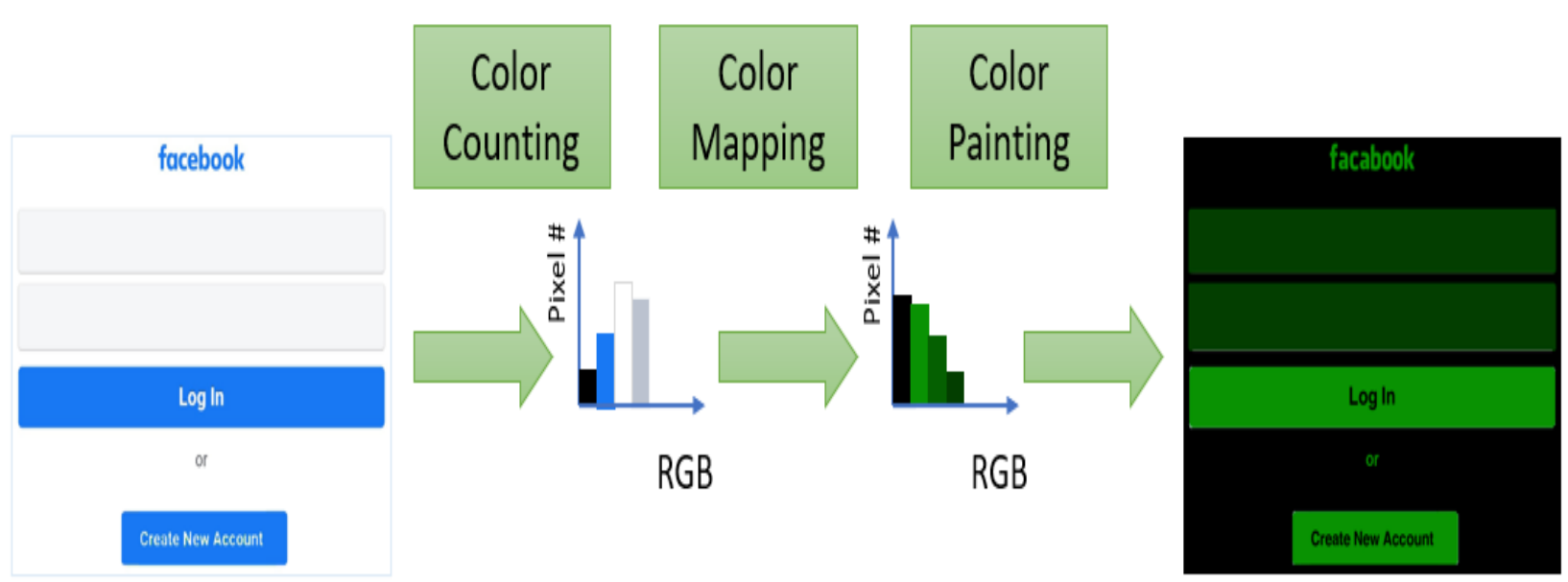

Figure 4. GUI objects' color transforming. [Adapted from reference 31]

In terms of video support, it is easy to make the video darker by putting a dark layer that has an opacity level on the video with the same size as it. As a result, power consumption will decrease and battery life will increase [31].

\subsection{Darkening OLED Display Partially}

As OLED displays depending on the colors that are being displayed, a new method was discovered to reduce the power consumption of OLED displays by darkening them partially. In this method, the black color which is known as a nonconsumer power color, active to darken the display by a specific ratio according to the OLED display. In other words, the pixels which include the diodes turn off.

This method made some experiments by analysing and modifying the android OS internal code. It is well known that when using mobile phones, a drawing process is being activated to show the new contents of the display which consumes more power, especially when scrolling the screen up and down [32]. By implementing this method, and by editing the source code of the Android operating system, it is possible to change the displaying parameters and the displaying dimensions according to the whole size of the OLED display, as it is related to the GPU drawing [33], by dividing the screen into the active and darkened screen as it is illustrated in Figure 5. In other words, it gets rid of the unnecessary computations which are happening in the android OS to view the new contents after scrolling up and down [16]. The study was made also by taking brightness into consideration, low and high brightness intensity, for both cases which are full display and partial display on OLED screens [16]. 


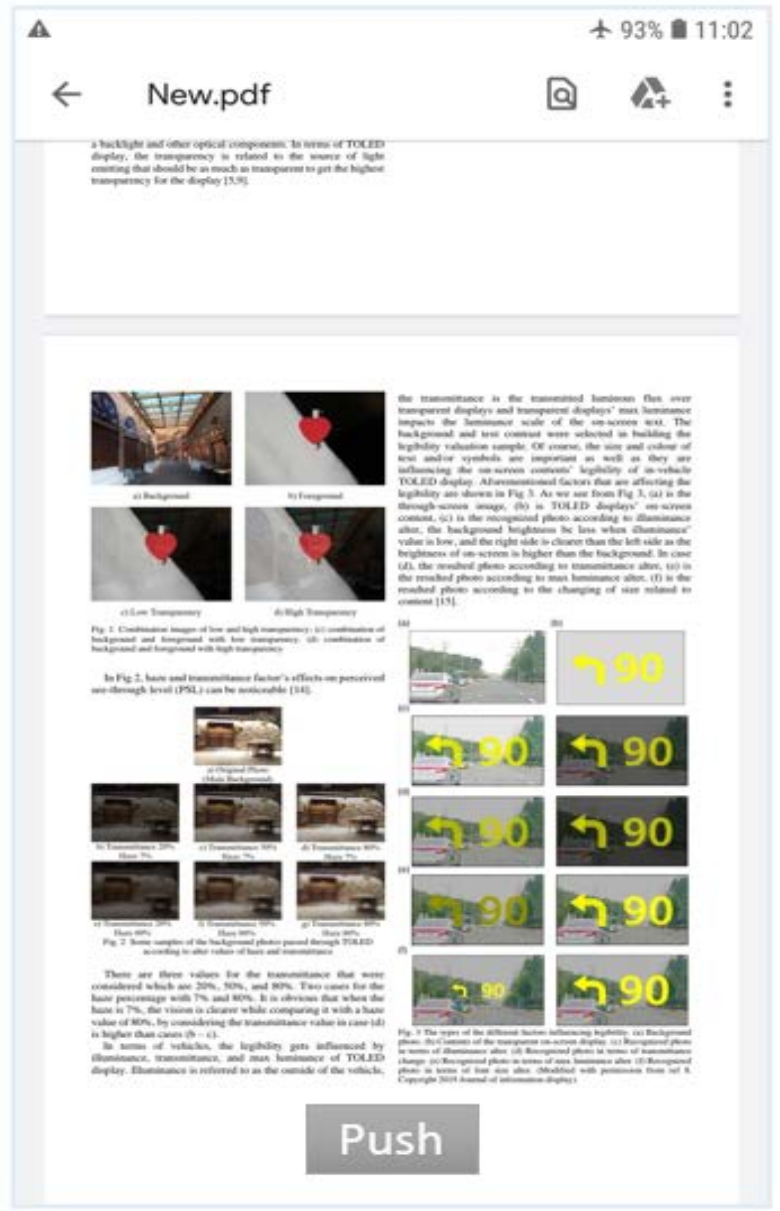

(a)

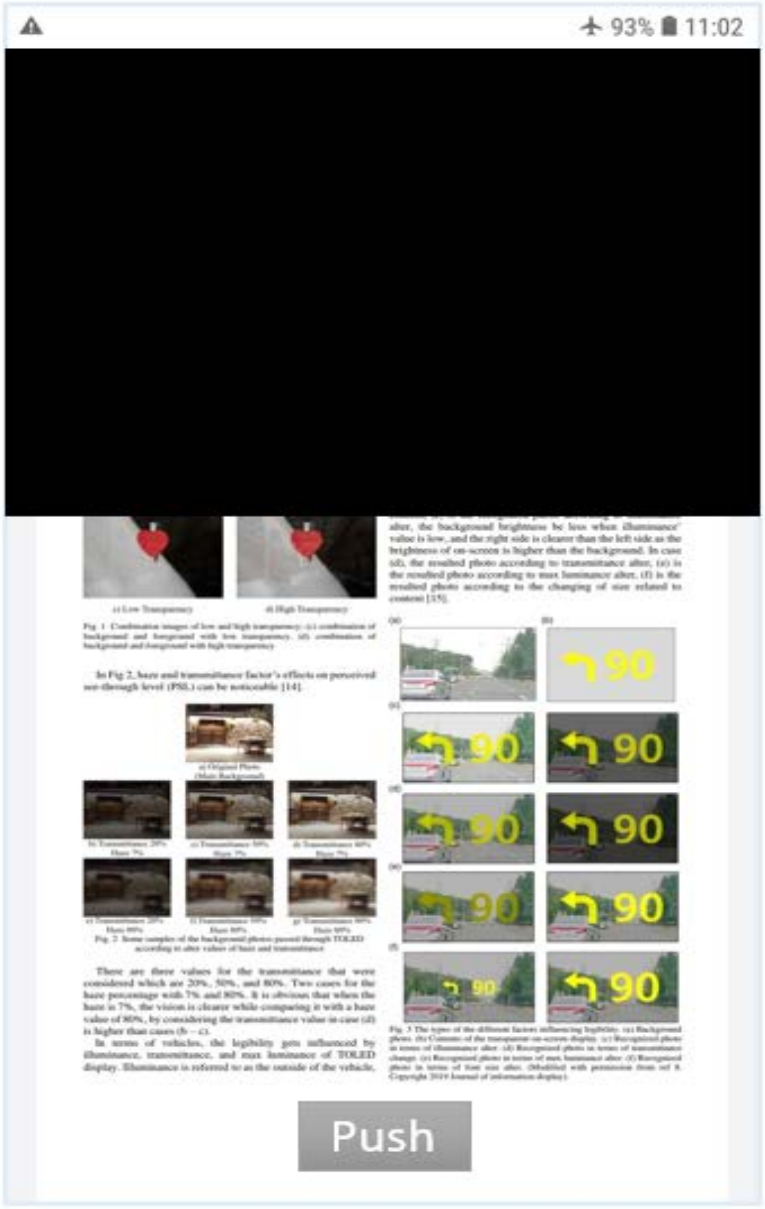

(b)

Figure 5. (a) Viewing full screen on whole OLED display (b) Viewing partial screen with $30 \%$ darkening display from top [Adapted from reference 16].

Some studies [34, 35, 36] concentrated on Dynamic Voltage Scaling (DVS) to reduce the power consumed by OLED display when showing a video as the model is dominate the OLED display’s input voltage.

\section{Results and Discussions}

The results of the unsupervised power-constrained contrast enhancement (PCCE) technique, region of interest (ROI) extraction \& region of noninterest (NON-ROI) Dimming method, and Chameleon method studies show in this section.

\subsection{Unsupervised PCCE Technique}

The conventional methods prevent selecting the requested (R) value, so they selected the parameters to get that value inversely, so the produced image gets the desired value of $(\mathrm{R}=0.5)$. When this value selects, power consumption reduces to its half as the intensity of the image's luminance reduces. As shown in Figure 6, the optical comparison is made between four methods, three of them are conventional and the fourth one is the proposed method. In Figure 6, (a) is the input image, (b) is HPCCE [4], (c) is Chang et al [37], (d) is Chondro et al [5], and (e) is the proposed method [15]. By comparing the produced images, we can easily notice that the proposed method has the best result by preserving the details of the image and improving the contrast for the fixed value of (R). The input image in Figure 6 (a) was taken from BSDS500 test sets of the dataset [38]. 


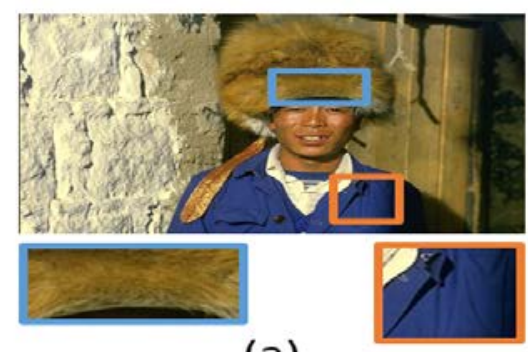

(a)

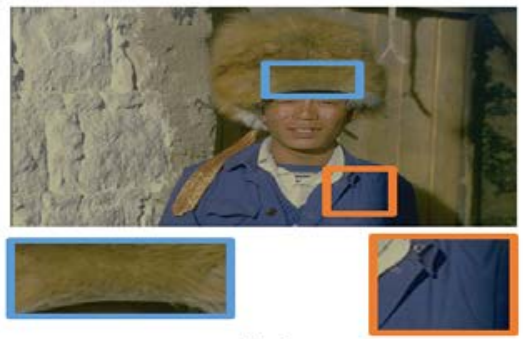

(b)

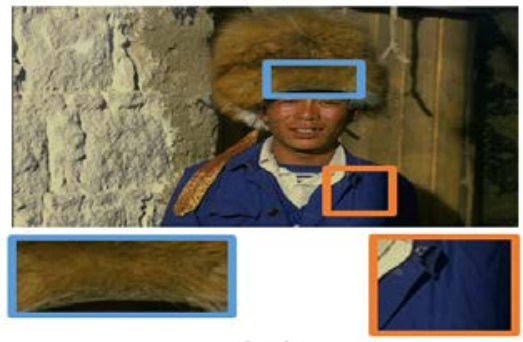

(d)

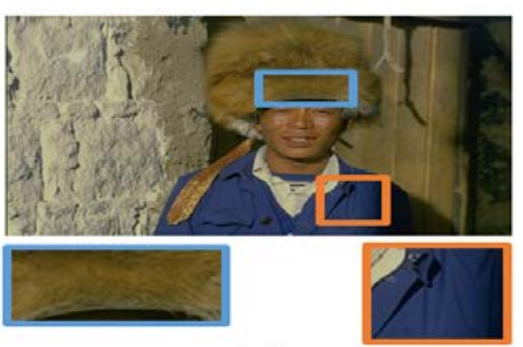

(c)

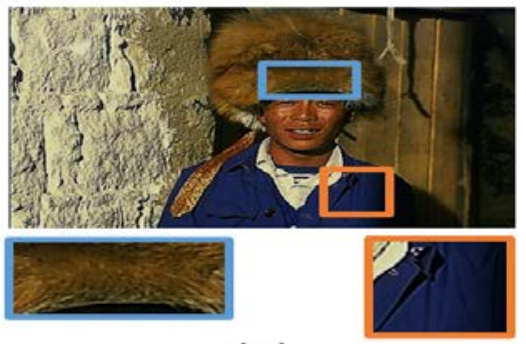

(e)

Figure 6. The optical comparison of the conventional and proposed method in BSDS500 test sets; (a) original photo, (b) HPCCE [4], (c) Chang et al [37], (d) Chondro et al [5], (e) proposed method [15] [Adapted from reference 15].

As mentioned before, the loss function is a combination of three factors, which are power loss $\mathrm{L}_{\mathrm{p}}$, similarity loss $\mathrm{L}_{\mathrm{s}}$, and contrast loss $\mathrm{L}_{\mathrm{c}}$. To check the performance of each factor, training was made on the ACE network without each factor. The proposed method is the best result which is the combination of the three factors is working together to get the superior image as it shows the high contrast, image details and at the same time limits the power consumption.

\subsection{Region Of Interest (ROI) Extraction \& Region Of Noninterest (NON-ROI) Dimming}

By using the proposed method of adjusting luminance \& saturation (ALS) and comparing it with the other two methods which are adjusting luminance (AL) and adjusting saturation (AS) in terms of power consumption, the best method selected for future usage. The previous methods applied to (Seagull) photos [8]. The result shows in Figure 7. The display model was selected to be the a $\mu$ OLED-32028-P1 AMOLED (P1 Active Matrix Organic Light Emitting Diode) with a power meter of HOIKI 3334. The resulted photo after implementing the three methods illustrates the differences between each of them in a visual way [8]. 


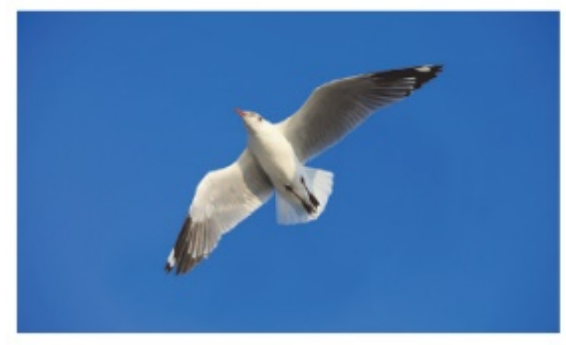

(a) Original

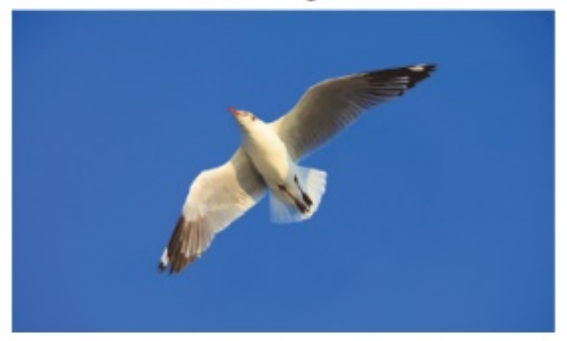

(c) AS

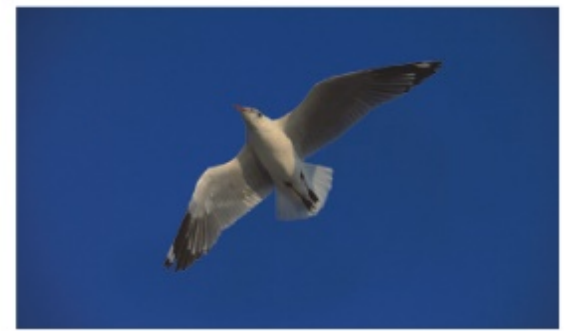

(b) $\mathrm{AL}$

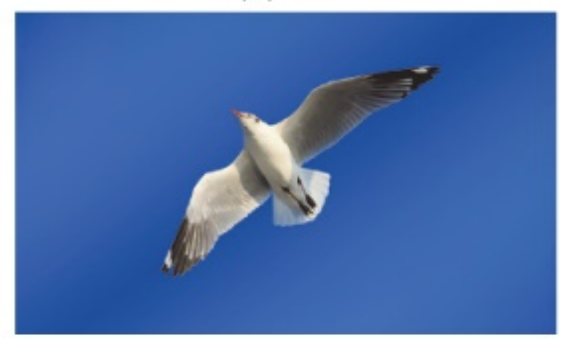

(d) ALS

Figure 7. Images processed by the three methods: (b) Using adjusting luminance, (c) Using adjusting saturation, (d) Using adjusting luminance \& saturation [Reproduced with permission from reference 8. Copyright 2016, Deguang Li et al. Mobile information systems].

When using adjust luminance method, the power reduced by a percentage of $26.68 \%$ in (b) case. In (c) case, the power decreased by $25.96 \%$. In the proposed method which is (d) case, power consumption of $26 \%$ reduced.

It was found that the adjusting luminance \& saturation method is effective in terms of saving power when the content of photos has blue or white colors with a high ratio. However, it is not much effective, when the major color of photos is grey.

\subsection{A Web Browser For Mobile OLED Displays; Chameleon}

Like every application, there are various options and modes to use and select from inside the program. Chameleon web browser offers four modes to select from which are; Dark, Green, Arbitrary, and Inversion mode as shown in Figure 8.
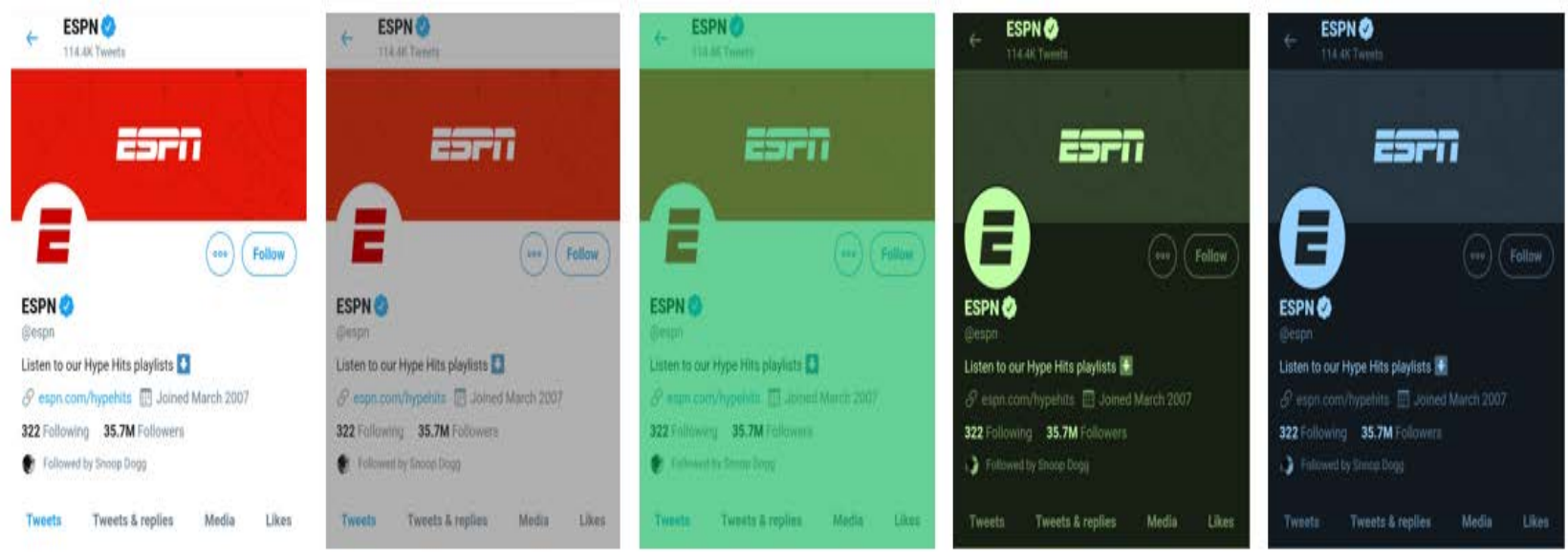

Figure 8. Original view and other four modes for displaying [Adapted from reference 31]. 
It was found that the power consumption decreased by $25 \%$ in dark mode, $34 \%$ in green mode, $66 \%$ in Inversion mode, and $72 \%$ in Arbitrary mode. Inversion mode uses blue color. In this result, the Arbitrary mode is the best in terms of saving power. Overall, and in this method, while surfing the internet and websites, $41 \%$ of the power consumption of the system was reduced.

\subsection{Darkening OLED Display Partially}

The presented method promises to achieve more by reducing the power consumption of OLED displays. By applying it on Nexus 6, and after shutting down some services like Bluetooth, Wi-Fi, GPS and putting it in airplane mode, the results came out. The method was able to reduce the power by $11.1 \%$ when low brightness was activated, and in high brightness mode the power reduction was $10.4 \%$.

\section{Conclusion}

Some studies were made to enhance saving power consumption for OLED displays. Energy-saving methods for OLED displays commonly used and information about the application of the methods is given in this review paper. This paper shows four various techniques which showed positive and promising results. The first work is by using an unsupervised deep learning method on PCCE with great results for the OLED display. This method is the convolutional neural network (CNN) which is used in the ACE network, and for training it. A new loss function is proposed which is a combination of three factors, power loss, similarity loss, and contrast loss. The result was superior in terms of contrast, details, and power consumption by comparing it with other methods. The second study depended on extracting edges and changing the values of luminance and saturation. The quality of photos was preserved and power consumption was reduced by $22.5 \%$. The third study was made by using a color adaptive web browser on mobile OLED devices, it showed a perfect performance in terms of power reduction which was reduced by $41 \%$ for the system power. The fourth and last study explained the idea of changing the computation of the drawing area of mobile OLED display devices, by altering the android operating system's code and getting rid of the unnecessary computation on the dimmed area. The reduction of power consumption reached $11.1 \%$ when the brightness was low, and $10.4 \%$ when high brightness was activated.

As a future work in terms of methods used, more advanced deep learning methodologies may be used to get more accurate results. In addition, some improvements could be done to the internet browser to make it more reliable by decreasing consumed power. Also, Android and iPhone systems' developers may possibly add some features that one of them is letting users darken a specific part of their phones' display by developing their internal system.

\section{References}

[1] S. Kang, "Image-Quality-Based Power Control Technique for Organic Light Emitting Diode Displays," in Journal of Display Technology, vol. 11, no. 1, pp. 104109, Jan. 2015, doi: https://10.1109/JDT.2014.2363086

[2] ALBABA, Mohammed; AKKUS, Meryem Sena. NEXT GENERATION DISPLAY TECHNOLOGY: TRANSPARENT ORGANIC LIGHT EMITTING DIODES. Selcuk-Teknik Dergisi, [S.l.], p. 168-180, dec. 2020. ISSN 1302-6178. Available at: http://sutod.selcuk.edu.tr/sutod/article/view/524. Date accessed: 29 Dec. 2020.

[3] C. Chae, H. Le, K. Lee, G. Cho and G. Cho, "A SingleInductor Step-Up DC-DC Switching Converter With Bipolar Outputs for Active Matrix OLED Mobile Display Panels," in IEEE Journal of Solid-State Circuits, vol. 44, no. 2, pp. 509-524, Feb. 2009, doi: https://10.1109/JSSC.2008.2010986

[4] C. Lee, C. Lee, Y. Lee and C. Kim, "Power-Constrained Contrast Enhancement for Emissive Displays Based on Histogram Equalization," in IEEE Transactions on Image Processing, vol. 21, no. 1, pp. 80-93, Jan. 2012, doi: https://10.1109/TIP.2011.2159387

[5] P. Chondro, C. Chang, S. Ruan and C. Shen, "Advanced Multimedia Power-Saving Method Using a Dynamic Pixel Dimmer on AMOLED Displays," in IEEE Transactions on Circuits and Systems for Video Technology, vol. 28, no. 9, pp. 2200-2209, Sept. 2018, doi: https://10.1109/TCSVT.2017.2723600

[6] A. Carroll and G. Heiser, "An analysis of power consumption in a smartphone," in Proceedings of the USENIX Conference on USENIX Annual Technical Conference, vol. 14, p. 21, Boston, Mass, USA, June 2010.

[7] X. Chen, Y.Chen, Z.Ma, andF. C. A. Fernandes, "How is energy consumed in smartphone display applications?" in Proceedings of the 14th Workshop on Mobile Computing Systems and Applications (ACM HotMobile '13), Jekyll Island, Georgia, USA, 26-27 February 2013.

[8] Deguang Li, Bing Guo, Yan Shen, Junke Li, Yanhui Huang, "Making Image More Energy Efficient for OLED Smart Devices", Mobile Information Systems, vol. 2016, Article ID 6575931, 8 pages, 2016. https://doi.org/10.1155/2016/6575931 
[9] S. Kim, S. Hyun, T. Heo, D. Im and J. Huh, "Blind: Power saving color transform method for OLED displays," 2016 IEEE International Conference on Consumer Electronics (ICCE), 2016, pp. 500-501, doi: https://10.1109/ICCE.2016.7430704

[10]M. Dong and L. Zhong, "Power Modeling and Optimization for OLED Displays," in IEEE Transactions on Mobile Computing, vol. 11, no. 9, pp. 1587-1599, Sept. 2012, doi: https://10.1109/TMC.2011.167

[11] Wee, Tan \& Balan, Rajesh. (2012) Adaptive Display Power Management for OLED Displays. ACM SIGCOMM Computer Communication Review. 42. $10.1145 / 2342480.2342487$

[12]R. Mertens, "Choosing color sets can reduce OLED power consumption by up to $40 \%$," [Online]. Jul 09, 2009, Available: https://www.oled-info.com/choosingcolor-sets-can-reduce-oled-power-consumption-40 Date accessed: 15 June. 2021

[13] A. Laaperi, "Disruptive Factors in the OLED Business Ecosystem,” Information Display Magazine, vol. 25, pp. 8-13, Sept. 2009.

[14] Minyoung Park and Minseok Song, "Saving Power in Video Playback on OLED Displays by Acceptable Changes to Perceived Brightness," J. Display Technol. 12, 483-490 (2016)

[15] Y. -G. Shin, S. Park, Y. -J. Yeo, M. -J. Yoo and S. -J. Ko, "Unsupervised Deep Contrast Enhancement With Power Constraint for OLED Displays," in IEEE Transactions on Image Processing, vol. 29, pp. 2834-2844, 2020, doi: https://10.1109/TIP.2019.2953352

[16] S. BoHum, K. Soontae. Enhancing Energy Efficiency on OLED Display Equipped Mobile Device.

[17]K. Zhang, W. Zuo, Y. Chen, D. Meng and L. Zhang, "Beyond a Gaussian Denoiser: Residual Learning of Deep CNN for Image Denoising," in IEEE Transactions on Image Processing, vol. 26, no. 7, pp. 3142-3155, July 2017, doi: https://10.1109/TIP.2017.2662206

[18] O. Oktay et al., "Anatomically Constrained Neural Networks (ACNNs): Application to Cardiac Image Enhancement and Segmentation," in IEEE Transactions on Medical Imaging, vol. 37, no. 2, pp. 384-395, Feb. 2018, doi: https://10.1109/TMI.2017.2743464

[19] Y. Nam, D. Choi and B. C. Song, "Power-Constrained Contrast Enhancement Algorithm Using Multiscale Retinex for OLED Display," in IEEE Transactions on Image Processing, vol. 23, no. 8, pp. 3308-3320, Aug. 2014, doi: https://10.1109/TIP.2014.2324288
[20]D. Jahier Pagliari, E. Macii and M. Poncino, "LAPSE: Low-Overhead Adaptive Power Saving and Contrast Enhancement for OLEDs," in IEEE Transactions on Image Processing, vol. 27, no. 9, pp. 4623-4637, Sept. 2018, doi: https://10.1109/TIP.2018.2844722

[21]P. Arbeláez, M. Maire, C. Fowlkes and J. Malik, "Contour Detection and Hierarchical Image Segmentation," in IEEE Transactions on Pattern Analysis and Machine Intelligence, vol. 33, no. 5, pp. 898-916, May 2011, doi: https://10.1109/TPAMI.2010.161

[22]F. Yu and V. Koltun, "Multi-Scale Context Aggregation by Dilated Convolutions," in International Conference on Learning Representations (ICLR), May 2016.

[23]D. Chen, J. Liao, L. Yuan, N. Yu and G. Hua, "Coherent Online Video Style Transfer," 2017 IEEE International Conference on Computer Vision (ICCV), 2017, pp. 11141123, doi: $\underline{\text { https://10.1109/ICCV.2017.126 }}$

[24]Z. Yi, H. Zhang, P. Tan and M. Gong, "DualGAN: Unsupervised Dual Learning for Image-to-Image Translation," 2017 IEEE International Conference on Computer Vision (ICCV), 2017, pp. 2868-2876, doi: https://10.1109/ICCV.2017.310

[25] Kingma, D.P., Ba, J.L., "Adam: a method for stochastic optimization". In: International Conference on Learning Representations, pp. 1-15 (2015).

[26] C. Lee, C. Lee, Y. Lee and C. Kim, "Power-Constrained Contrast Enhancement for Emissive Displays Based on Histogram Equalization," in IEEE Transactions on Image Processing, vol. 21, no. 1, pp. 80-93, Jan. 2012, doi: https://10.1109/TIP.2011.2159387

[27] L. Ding andA.Goshtasby, "On the canny edge detector," Pattern Recognition, vol. 34, no. 3, pp. 721-725, 2001. ISSN 0031-3203, doi: https://doi.org/10.1016/S00313203(00)00023-6

[28] T. K.Wee andR. K. Balan, "Adaptive display power management for OLED displays," in Proceedings of the 1st ACM SIGCOMM Workshop on Mobile Gaming (MobiGames '12), pp. 25-30, Helsinki, Finland, August 2012. doi: https://doi.org/10.1145/2377677.2377770

[29]X. Chen, Y. Chen and Chun Jason Xue, "DaTuM: Dynamic tone mapping technique for OLED display power saving based on video classification," 2015 52nd ACM/EDAC/IEEE Design Automation Conference (DAC), 2015, pp. 1-6, doi: https://10.1145/2744769.2744814

[30] Mian Dong, Y. K. Choi and L. Zhong, "Power modeling of graphical user interfaces on OLED displays," 2009 
46th ACM/IEEE Design Automation Conference, 2009, pp. 652-657.

[31] M. Dong and L. Zhong, "Chameleon: A Color-Adaptive Web Browser for Mobile OLED Displays," in IEEE Transactions on Mobile Computing, vol. 11, no. 5, pp. 724-738, May 2012, doi: 10.1109/TMC.2012.40. https://www.sigmobile.org/mobisys/2011/program.html

[32] J. Yu et al., "Sensing Human-Screen Interaction for Energy-Efficient Frame Rate Adaptation on Smartphones," in IEEE Transactions on Mobile Computing, vol. 14, no. 8, pp. 1698-1711, 1 Aug. 2015, doi: https://10.1109/TMC.2014.2352862

[33] Android Developers. Hardware acceleration. [Online]. Available: https://developer.android.com/guide/topics/graphics/har dware-accel.html Date accessed: 19 Nov. 2020

[34] Xiang Chen, Jian Zeng, Yiran Chen, Wei Zhang and Hai $\mathrm{Li}$, "Fine-grained dynamic voltage scaling on OLED display," 17th Asia and South Pacific Design Automation Conference, 2012, pp. 807-812, doi: https://10.1109/ASPDAC.2012.6165066

[35] X. Chen, J. Zheng, Y. Chen, M. Zhao and C. J. Xue, "Quality-retaining OLED dynamic voltage scaling for video streaming applications on mobile devices," DAC Design Automation Conference 2012, 2012, pp. 10001005.

[36]B.-H. Lee and Y.-J. Kim, “Dynamic Voltage Scaling Using Scene Change Detection for Video Playback on Mobile AMOLED Displays,” in Proceedings of the 2016 International Symposium on Low Power Electronics and Design. Association for Computing Machinery, New York, NY, USA, 2016, pp. 302-307. doi: https://doi.org/10.1145/2934583.2934640

[37] T. Chang, S. S. Xu and S. Su, "SSIM-Based Quality-onDemand Energy-Saving Schemes for OLED Displays," in IEEE Transactions on Systems, Man, and Cybernetics: Systems, vol. 46, no. 5, pp. 623-635, May 2016, doi: https://10.1109/TSMC.2015.2455020

[38]P. Arbeláez, M. Maire, C. Fowlkes and J. Malik, "Contour Detection and Hierarchical Image Segmentation," in IEEE Transactions on Pattern Analysis and Machine Intelligence, vol. 33, no. 5, pp. 898-916, May 2011, doi: https://10.1109/TPAMI.2010.161 